\title{
Retraction Note to: Riding on Air: A New Theory for Lift Mechanics of Downhill Skiing and Snowboarding
}

\section{QianhongWu,YesimIgci,YiannisAndrepoulosand SheldonWeinbaumet al.}

RetractionNote to: QianhongWu,YesimIgci,YiannisAndrepoulosand SheldonWeinbaum et al., Riding on Air: A New Theory for Lift

Mechanics of Downhill Skiing and Snowboarding,

DOI: 10.1007/978-0-387-46050-5_50

The Editors have retracted this Conference Paper because the content overlaps significantly with a previously published article (Wu et al., 2006). Qianhong Wu does not agree with this retraction. Yiannis Andreopoulos and Sheldon Weinbaum have not responded to correspondence about this retraction. The Editors could not obtain a current email address for Yesim Igci. Wu, Q., Igci, Y., Andreopoulos, Y., \& Weinbaum, S. (2006) Lift mechanics of downhill skiing and snowboarding. Medicine and Science in Sports and Exercise, 38(6), 1132-1146. 